\title{
ASYMPTOMATIC CARRIAGE OF ANTIBIOTIC- RESISTANT GRAM-POSITIVE COCCI AMONG DIFFERENT BACKGROUND POPULATIONS IN EAST TIMOR, SOUTHEAST ASIA
}

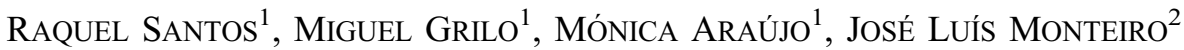 \\ and MANUELA OLIVEIRA ${ }^{1 *}$ \\ ${ }^{1}$ CIISA - Centre for Interdisciplinary Research in Animal Health, Faculty of Veterinary \\ Medicine, University of Lisbon, Lisbon, Portugal \\ ${ }^{2}$ OIKOS - Cooperação e Desenvolvimento, Oeiras, Portugal
}

(Received: 1 May 2018; accepted: 28 May 2018)

\begin{abstract}
Dissemination of pathogenic multiresistant bacteria is of public health concern. Reliable data can be difficult to obtain, especially in developing countries. This work aimed to characterize the skin and oropharyngeal microbiota, as well as their antimicrobial resistance profiles, of East-Timor populations to identify potentially pathogenic Gram-positive cocci. In order to assess the prevalence of pathogenic bacteria in East-Timor, the oropharyngeal and skin microbiota of 74 individuals was characterized. Gram-positive cocci were evaluated and their antimicrobial resistance profiles were determined. A total of 228 oropharyngeal and 278 skin samples were obtained. The population consisted of $36.5 \%$ of asymptomatic carriers of Grampositive cocci. Kocuria rosea $(n=7,19.4 \%)$, Staphylococcus spp. $(n=6,16.7 \%)$, and Micrococcus luteus $(n=6,16.7 \%)$ were isolated, among others. Antimicrobial resistance levels ranged between $0 \%$ and $36.1 \%$, and a multiresistance profile was observed in one third of the isolates. Gram-positive cocci colonization was associated with age group. Prevalence of multiresistant isolates was higher in males who were sampled at the refugee camp. Results show that the prevalence of antimicrobial resistance on East Timor may be underestimated. This study represents the first step toward the full characterization of potential pathogenic Gram-positive cocci present in the populations from East Timor.
\end{abstract}

Keywords: antimicrobial resistance, East Timor, Gram-positive cocci, oropharynx, skin

*Corresponding author; E-mail: moliveira@fmv.ulisboa.pt 


\section{Introduction}

The oropharynx and skin are epithelial surfaces constantly exposed to microorganisms, being important sites for pathogen colonization. Gram-positive cocci include several commensal genera and also important human pathogens. Streptococci and staphylococci, in particular, are a major threat to human health, since they can cause severe invasive infections. It is estimated that they are responsible for at least one third of all bacterial infections of humans, including tonsillitis, pneumonia, otitis media, meningitis, food poisoning, skin diseases, and septic shock [1].

These potentially pathogenic bacterial groups can live as commensals in individuals with a competent immune system, which may allow horizontal spreading within the community, or be responsible for transient or persistent opportunistic infections [2, 3]. For that reason, independently of the occurrence of an infection, it is extremely important to monitor the prevalence and distribution of this potentially pathogenic bacterial group and to determine its antimicrobial resistance profile [4]. In fact, Gram-positive bacteria often present high levels of antimicrobial resistance $[5,6]$. Emergence of resistant bacteria is generally attributed to selective pressure due to the excessive use or misuse of antimicrobial compounds [6]. Resistant pathogen dissemination routes must be considered for the establishment of adequate measures for treatment and prevention of infectious diseases in humans [7].

A comprehensive knowledge of the human microbiota begins with in-depth surveys of the bacterial community present in each individual. Identification and characterization of the antimicrobial resistance profile of this microbiota provides fundamental information for the monitorization of possible pathogens present in asymptomatic carriers. These data may provide relevant information about the epidemiological situation and the occurrence of resistance determinants that spread in developing countries, where the use and access to antimicrobial compounds are scarce.

This work aimed to characterize the skin and oropharyngeal microbiota of East Timor populations, to identify potentially pathogenic Gram-positive cocci and to determine their antimicrobial resistance profiles. Samples were obtained from diversified population groups, including both genders, a large range of ages, and different socioeconomic status.

\section{Methods}

Participants and data collection

A total of 148 swab samples were collected from 74 healthy individuals in East Timor, Southeast Asia, between July and August 2006, including skin $(n=74)$ and oropharyngeal swabs $(n=74)$. Samples were collected from three 
Table I. Characteristics of the sampled populations: location, gender, and age group

\begin{tabular}{lccccccc}
\hline & \multicolumn{7}{c}{ No. of samples collected } \\
\cline { 3 - 7 } & & & \multicolumn{5}{c}{ Age group (years) } \\
\cline { 3 - 7 } Location & Males & Females & $2-10$ & $11-20$ & $21-30$ & $31-40$ & $41-49$ \\
\hline Nairete & 11 & 15 & 16 & 3 & 2 & 4 & 1 \\
Refugee camp & 22 & 12 & 31 & 3 & 0 & 0 & 0 \\
Dili household & 9 & 5 & 6 & 3 & 0 & 2 & 3 \\
Total $(n=74)$ & 42 & 32 & 53 & 9 & 2 & 6 & 4 \\
\hline
\end{tabular}

distinct locations: a household and a refugee camp in Dili and in Nairete, a small rural village. Sampled population was composed by males $(n=42)$ and females $(n=32)$ who were aged between 2 and 49 years, as discriminated in Table I.

After collection, Amies swabs (Oxoid Ltd., Hampshire, UK) were then kept refrigerated until transported to our laboratory, where they were further processed.

\section{Bacterial isolation and phenotypic identification}

Skin and oropharyngeal swab samples were plated onto Columbia agar plates supplemented with 5\% sheep blood (COS, bioMérieux, Marcy l'Etoile, France) and incubated aerobically at $37^{\circ} \mathrm{C}$ for $24-48 \mathrm{~h}$. A total of 506 isolates were obtained through conventional microbiological methods and characterized through their macro- and microscopic morphologies and staining and biochemical characteristics (catalase and oxidase). Gram-positive cocci $(n=36)$ were further identified using biochemical identification galleries: API ID 32 Staph $^{\circledR}$ and API 20 Strep $^{\mathbb{R}}$ (bioMérieux), according to the manufacturer's instructions. Isolates were maintained at $-80^{\circ} \mathrm{C}$ until further characterization.

\section{Antimicrobial resistance profiling}

Antimicrobial resistance profile of the isolates was determined using the following nine compounds that are commonly used in human medicine and directed toward different bacterial targets: vancomycin (VA; $30 \mu \mathrm{g}$ ), amikacin (AK; $30 \mu \mathrm{g}$ ), penicillin $\mathrm{G}$ (P; 10 units), tetracycline (TE; $30 \mu \mathrm{g}$ ), erythromycin (ERY; $15 \mu \mathrm{g}$ ), gentamicin (CN; $10 \mu \mathrm{g}$ ), clindamycin (DA; $2 \mu \mathrm{g}$ ), sulfamethoxazole/ trimethoprim 19:1 (SXT; $25 \mu \mathrm{g}$ ), and norfloxacin (NOR; $10 \mu \mathrm{g}$ ) (Table II). Antimicrobial disks were purchased from Oxoid Ltd. Susceptibility to the compounds was tested by the disk diffusion method, as recommended by the Clinical and Laboratory Standards Institute guidelines [8]. 


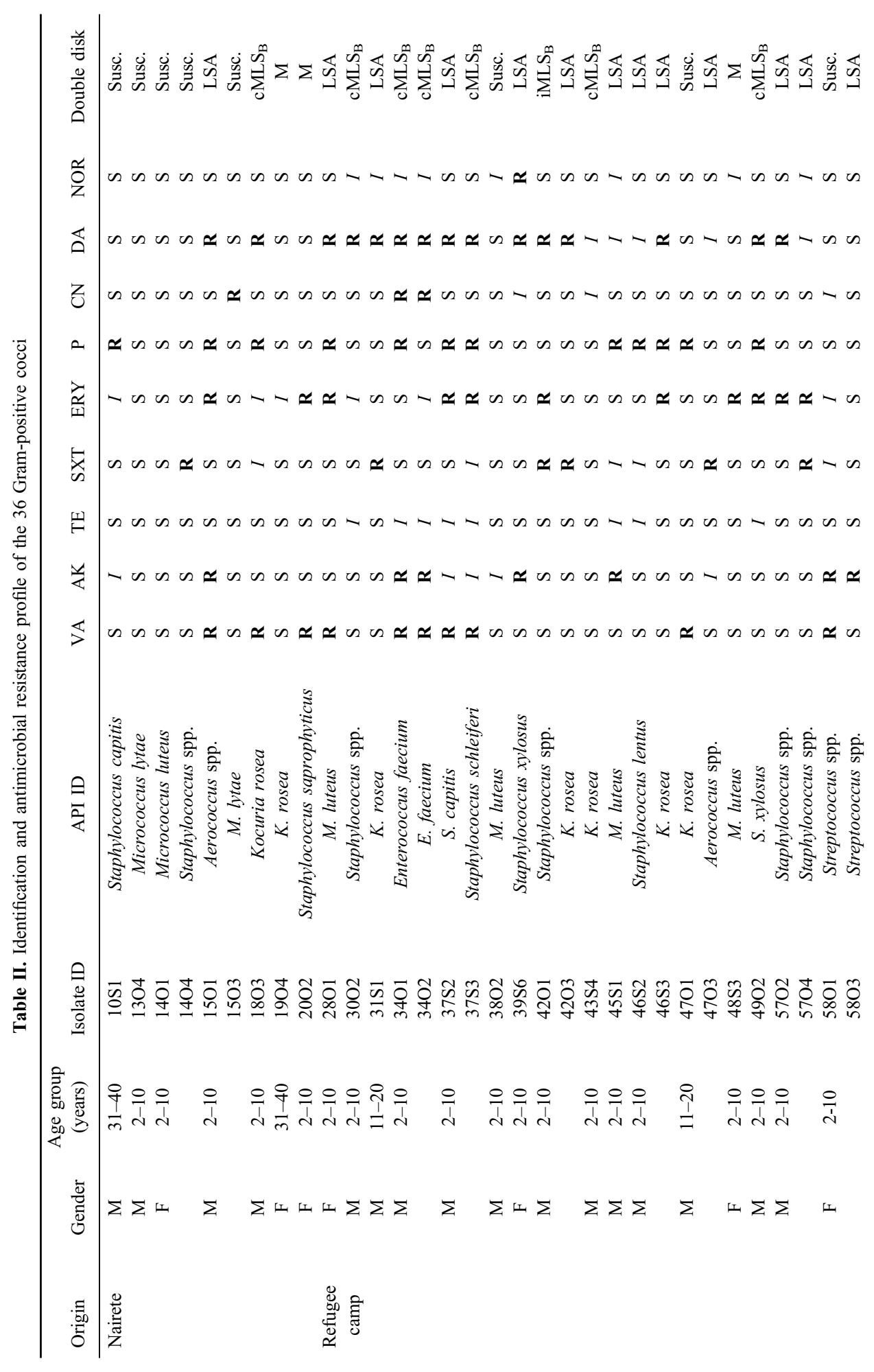




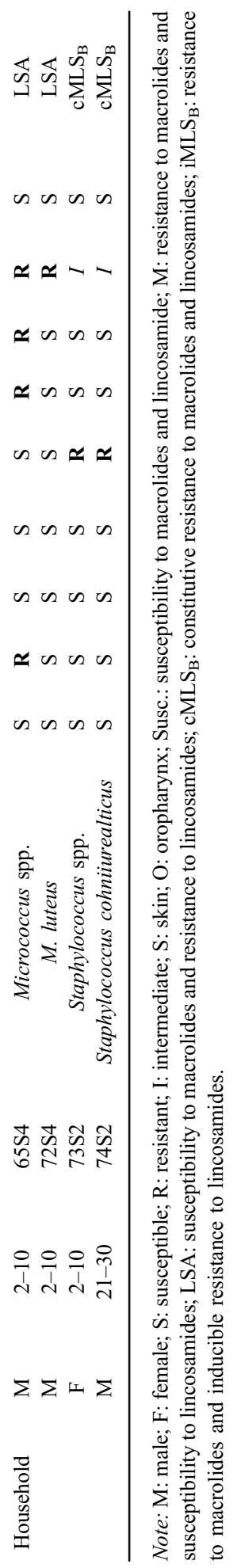


In addition, to determine the macrolide resistance profile, a double-disk test was performed using the antimicrobial compounds ERY and DN. Mueller-Hinton agar (bioMérieux) plates were inoculated and both disks were placed in the center with a $15-\mathrm{mm}$ distance. Plates were then incubated aerobically at $37^{\circ} \mathrm{C}$ for $24 \mathrm{~h}$. Formation of inhibition halos allows the isolates classification into five possible macrolide resistance phenotypes as follows: Susc., susceptibility to macrolides (ERY) and lincosamides (DA); M, resistance to macrolides and susceptibility to lincosamides; LSA, susceptibility to macrolides and resistance to lincosamides; $\mathrm{cMLS}_{\mathrm{B}}$, constitutive resistance to macrolides and lincosamides; and $\mathrm{iMLS}_{\mathrm{B}}$, resistance to macrolides and inducible resistance to lincosamides. The $\mathrm{cMLS}_{\mathrm{B}}$ phenotype can be identified when an inhibition halo does not occur around the ERY nor the DA disks. The $\mathrm{iMLS}_{\mathrm{B}}$ phenotype can be identified when an inhibition halo does not appear around the ERY, but a distorted (D-shaped) inhibition halo appears around the DA disk.

\section{Statistical analysis}

In order to test the independence between the prevalence of Gram-positive cocci and multiresistant isolates with sociobiological data (age group, gender, and origin), a $\chi^{2}$ test was performed. A log-linear model was further applied to identify dependencies between the prevalence of multiresistant isolates, gender, age group, and individual origin. The analysis of standardized residuals was used to identify cells with bad adjustments. Standardized residuals which were greater than 1.96 (in absolute value) were considered to be significantly different from 0 for a probability of type 1 error of 0.05 . Statistical significance was considered at $p<0.05$. All statistical analysis were performed using IBM SPSS Statistics ${ }^{\circledR}$, version 24 (IBM Analytics, New York, USA).

\section{Statement of human and animal rights}

In this study, no experimental research was conducted on vertebrates or any regulated invertebrates. This research was reviewed and approved by the Faculty of Veterinary Medicine, University of Lisbon.

\section{Results}

Bacterial isolation and phenotypic identification

From the oropharyngeal samples, 228 isolates were obtained, including Gram-positive cocci (16 catalase-positive and 6 catalase-negative isolates), 
Gram-positive bacilli (76 catalase-positive and 28 catalase-negative isolates), and Gram-negative bacilli (55 oxidase-positive and 47 oxidase-negative isolates). From the skin samples, it was possible to obtain 278 isolates, including Gram-positive cocci (14 catalase-positive and 1 catalase-negative isolates), Gram-positive bacilli (172 catalase-positive and 14 catalase-negative isolates), and Gram-negative bacilli (61 oxidase-positive and 16 oxidase-negative isolates).

Gram-positive cocci were selected for further analysis, due to their relevance as potentially pathogenic microorganisms and their role in antimicrobial resistance dissemination. Evaluation of results concerning age, gender, and socioeconomic status revealed that $36.5 \%(n=27)$ of the population were asymptomatic carriers of Gram-positive cocci. This prevalence was higher in children aged between 2 and 10 years, reaching 29.7\% $(n=22)$. On the other hand, only $6.7 \%(n=5)$ of the population older than 11 years were carriers of Gram-positive cocci. Individuals from the refugee camp presented the higher prevalence of Gram-positive cocci $(n=16,21.6 \%)$, followed by the ones from Nairete and the Dili household with a prevalence of $9.5 \%(n=7)$ and $5.4 \%$ $(n=4)$, respectively. Almost half of the male population carried Gram-positive cocci $(n=19,25.7 \%)$, whereas in females, only $10.8 \%(n=8)$ were found to be carriers.

The results of the biochemical identification of Gram-positive cocci by API ID $32 \mathrm{Staph}^{\circledR}$ and API $20 \mathrm{Strep}^{\circledR}$ (bioMérieux) are shown in Table II. Most prevalent genera were Staphylococcus $(n=14,38.9 \%)$ [Staphylococcus spp. ( $n=6,16.7 \%), S$. capitis $(n=2,5.6 \%), S$. xylosus $(n=2,5.6 \%), S$. cohniiurealticus $(n=1,2.8 \%)$, S. lentus $(n=1,2.8 \%), S$. saprophyticus $(n=1,2.8 \%)$, S. schleiferi $(n=1,2.8 \%)]$, Micrococcus $(n=9,25 \%)$ [M. luteus $(n=6,16.7 \%)$, Micrococcus lylae $(n=2,5.6 \%)$, Micrococcus spp. $(n=1,2.8 \%)]$, and Kocuria $(n=7,19.4 \%)$ $[K$. rosea $(n=7,19.4 \%)]$. Isolates belonging to Streptococcus $(n=2,5.6 \%)$ [Streptococcus spp. $(n=2,5.6 \%)]$, Enterococcus $(n=2,5.6 \%)$ [E. faecium $(n=2,5.6 \%)]$, and Aerococcus $(n=2,5.6 \%)$ [Aerococcus spp. $(n=2,5.6 \%)$ ] genera were also obtained.

\section{Antimicrobial resistance testing}

The antimicrobial resistance profile of the Gram-positive cocci $(n=36)$ is presented in Table II. None of the Gram-positive isolates was resistant to TE. Low levels of resistance were observed for NOR $(n=1,2.8 \%), \mathrm{CN}(n=2,5.6 \%)$, and SXT $(n=6,16.7 \%)$, whereas intermediate levels of resistance were observed for other tested antimicrobials, such as AK $(n=8,22.2 \%)$, VA $(n=10,27.8 \%)$, ERY $(n=13,36.1 \%)$, and $\mathrm{P}(n=13,36.1 \%)$. The highest level of resistance was observed regarding DA $(n=17,47.2 \%)$. 
It was observed that one third of the Gram-positive cocci $(n=10,27.7 \%)$ presented a multiresistance profile, which is characterized by resistance to more than three classes of antimicrobial compounds.

The double-disk test for macrolide resistance characterization revealed that only $22.2 \%(n=8)$ of the isolates presented the $\mathrm{S}$ phenotype. Remaining isolates presented the $\mathrm{iMLS}_{\mathrm{B}}(n=1,2.7 \%), \mathrm{M}(n=3,8.3 \%), \mathrm{cMLS}_{\mathrm{B}}(n=9,25 \%)$, and LSA $(n=15,41.7 \%)$ phenotypes (Table II).

Evaluation regarding age, gender, and socioeconomic status showed that males presented higher resistance levels for DA and P, whereas females presented higher resistance for ERY, VA, and AK (Figure 1). Children from the 2-10 years age group presented the highest multidrug resistance levels (Table II). Nairete population presented higher levels of resistance for VA and $\mathrm{P}$, the refugee camp population for DA, and the Dili Household population for DA and ERY (Figure 2).

\section{Statistical analysis}

Analysis revealed that colonization with Gram-positive cocci in children in the age group of $2-10$ years $\left[\chi^{2}(1)=4.331 ; p=0.037\right]$, as well as the prevalence of multiresistant isolates in individuals who were located to the refugee camp $\left[\chi^{2}(1)=\right.$ 7.608; $p=0.006$ ], were statistically significant. Furthermore, log-linear analysis showed a dependency between variables regarding prevalence of multiresistant isolates, gender, and origin of individuals $\left[\chi^{2} \mathrm{~L}(7)=13.784 ; p=0.037\right]$. Standardized residual analysis showed that multiresistant isolate prevalence was significantly associated with male individuals who were sampled in the refugee camp.

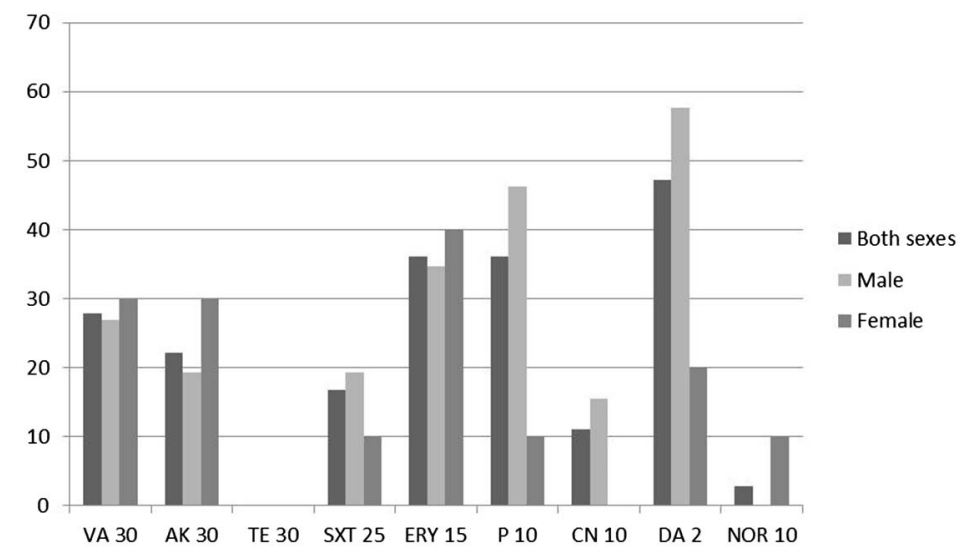

Figure 1. Antimicrobial resistance distribution according to gender 


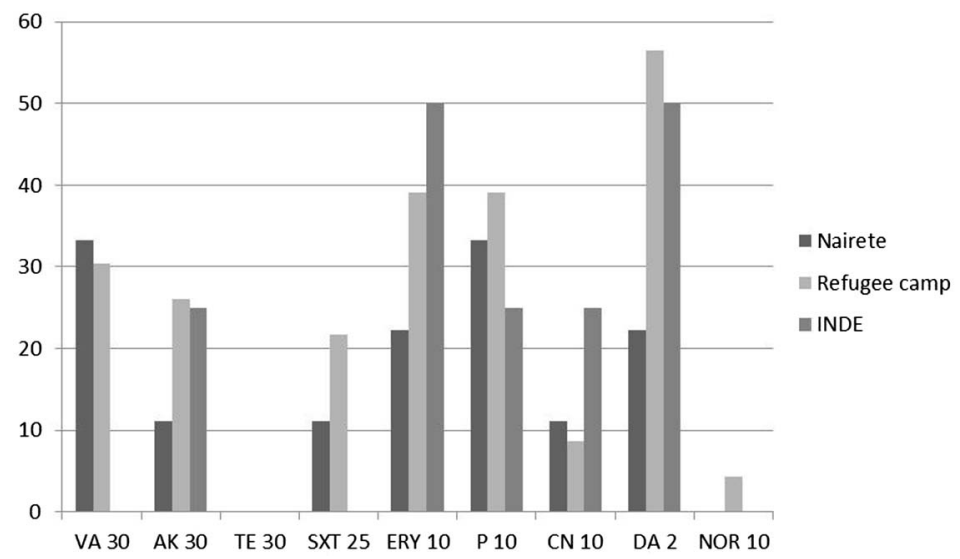

Figure 2. Antimicrobial resistance distribution according to geographical location

\section{Discussion}

In recent years, there has been a growing concern about antimicrobial resistance dissemination. Epidemiological studies on the microbiota of asymptomatic populations are especially relevant, since they allow the detection of potentially pathogenic bacteria. However, such reports on developing countries are extremely scarce.

This study aimed to characterize the skin and oropharynx microbiota and its antimicrobial resistance profile of East Timor individuals. Three distinct populations were sampled: two located in Dili, the city capital of East-Timor, and one in Nairete. The first sampled population in Dili was composed by internally displaced people from a refugee camp, with a population of more than 400 individuals who were living in extremely close quarters on tends, within the boundaries of an empty pharmaceutical warehouse. Habitants ran away or lost their homes and belongings due to violence occurring between different groups in the capital. Food supply was scarce and irregular, complemented by the support of international organizations. The camp had only two water supply sources, and the waste disposal system was severely overwhelmed by the large number of people present. Health care was almost inexistent.

The other population sampled in Dili was composed by individuals belonging to a single household with access to running water, energy, stable supply of food and health care, including antimicrobial compounds, when necessary.

Nairete is a small rural village, being the mostly Eastern province of East Timor. It mainly depends on agriculture. The village has a relatively stable food supply and is known as one of the most promising villages in the area, benefiting 
from being close to Lospalos, the region capital. It has no running water and uses traditional means of waste disposal. Water is abundant, with the village sitting on top of a small river. It has a very small health station, but access to pharmaceuticals is scarce.

Based on conventional microbiological procedures, bacterial isolation from the sampled populations originated a total of 506 isolates. Gram-positive bacteria were the most prevalent (64.6\%), although a high percentage of Gram-negative $(35.4 \%)$ was also observed. Since this bacterial group is very susceptible to environmental conditions, the high percentage of Gram-negative isolates seems to indicate that sample collection and transport were performed in adequate conditions [9].

For subsequent analysis, a group of 36 Gram-positive cocci was selected, including Micrococcus $(n=9)$, Kocuria $(n=7)$, Streptococcus $(n=2)$, Enterococcus $(n=2)$, and Aerococcus $(n=2)$, and due to their relevance as potentially pathogenic microorganisms and their role in the transmission of antimicrobial resistance determinants [5]. However, since sampled individuals were apparently healthy, showing no gross signs of disease, they were classified as asymptomatic carriers.

Regarding isolates distribution among different genders, age groups, and geographic location, it was observed that children up to 10 years old presented a higher relative frequency $(41.5 \%)$ of Gram-positive cocci, whereas on the remaining population only $23.8 \%$ of the individuals were asymptomatic carriers of this bacterial group. The statistical association was evident in the age group of 2-10 years. Young children present specific characteristics, including a developing immune system and an increased contact with environmental microbiota, rendering them more susceptible to bacterial colonization [3]. The higher contact with potentially contaminated environments can also explain the higher prevalence of Gram-positive cocci in males $(45.2 \%)$ than in females $(25 \%)$.

Socioeconomic constraints may have an important role in the dissemination of potentially pathogenic bacteria, as suggested by the higher prevalence $(47.1 \%)$ in the refugee camp population. This population lived in extremely poor conditions with scarce food and water supplies. Moreover, the waste disposal system was almost inexistent and people did not have adequate hygiene conditions. Considering their extremely difficult situation, these prevalence results were not surprising. In this study, this hypothesis is further corroborated by the association of multiresistant isolates with males who were sampled in the refugee camp.

Antimicrobial-resistant bacteria are a major concern. In this study, TE was the only antimicrobial to which all isolates were susceptible, while the highest resistance levels were obtained for DA (47.2\%) and ERY and P (36.1\%). 
Resistance in Gram-positive cocci has increased dramatically worldwide, particularly to macrolides $[10,11]$, as corroborated by the fact that $77.8 \%$ of the isolates studied presented a resistance profile to these drugs. This is in accordance with the earlier studies that demonstrated that the resistance to macrolides (such as ERY) and lincosamides (such as DA) is prevalent among Gram-positive cocci [12-15].

Emergence, selection, and dissemination of resistant bacteria are mainly attributed to the selective pressure of antimicrobials misuse and abuse [16, 17]. Thus, it was expected that the individuals from the Dili household, who have improved socioeconomic conditions, access to health care system and pharmaceuticals, would present a higher prevalence of antimicrobial-resistant bacteria. However, our results showed that the prevalence among all three sampled populations was similar. As antimicrobial drugs administration was extremely rare in the refugee camp and in the Nairete village, multiresistance levels observed might be originated from environmental contamination. In fact, the refugee camp was located within the boundaries of an empty pharmaceutical warehouse, while in the Nairete region, antimicrobial resistance could have disseminated due to the presence of international soldiers during the 30 years of East Timor occupation, which had access to antimicrobial drugs. Salyers and Shoemaker [18] showed that resistant bacteria and antimicrobial compounds can travel through the ground water originated from human wastes, entering environments where no antibiotics are being administrated directly to the populations, supporting our theory. In addition, soldiers proceeded to perform intense fumigation, which may also contributed to the high level of antimicrobial resistance observed. In fact, references to non-antibiotic selection pressure due to heavy metals or disinfectants have already been described [7].

Identification of skin and oropharyngeal microbiota is an important first step toward the whole characterization of potential pathogenic bacteria of developing populations. Our results suggest that drug resistance may be very significant in these countries and should be monitored to provide accurate information on antimicrobial resistance prevalence. Furthermore, risk factors detected in this study, such as a higher prevalence of multiresistant isolates at the refugee camp, should be considered in the development of appropriate health management practices in emergency situations.

\section{Acknowledgements}

This work would not be possible without the support of Professor Cristina Lobo Vilela, 1958-2013, and Professor Ilda Sanches, 1958-2017. Work was supported by CIISA - Centre for Interdisciplinary Research in Animal Health, 
Faculty of Veterinary Medicine, University of Lisbon, funded by Project UID/CVT/276/2013 (CIISA).RS, MG, and MA have contributed equally to this work.

\section{Conflict of Interest}

None.

\section{References}

1. Nitsche-Schmitz, D., Rohde, M., Chhatwal, G.: Invasion mechanisms of Gram-positive pathogenic cocci. Thromb Haemost 98, 488-496 (2007).

2. Bogaert, D., De Groot, R., Hermans, P. W.: Streptococcus pneumoniae colonization: The key to pneumococcal disease. Lancet Infect Dis 4, 144-154 (2004).

3. Jourdain, S., Smeesters, P. R., Denis, O., Dramaix, M., Sputael, V., Malaviolle, X., Van Melderen, L., Vergison, A.: Differences in nasopharyngeal bacterial carriage in preschool children from different socio-economic origins. Clin Microbiol Infect 17, 907-914 (2011).

4. Kaszanyitzky, E. J., Jánosi, S., Egyed, Z., Agost, G., Semjén, G.: Antibiotic resistance of staphylococci from humans, food and different animal species according to data of the Hungarian resistance monitoring system in 2001. Acta Vet Hung 51, 451-464 (2003).

5. Rice, L. B.: Antimicrobial resistance in Gram-positive bacteria. Am J Med 119, S11-S19 (2006).

6. Weigel, L. M., Donlan, R. M., Shin, D. H., Jensen, B., Clark, N. C., McDougal, L. K., Zhu, W., Musser, K. A., Thompson, J., Kohlerschmidt, D., Dumas, N., Limberger, R. J., Patel, J. B.: High-level vancomycin-resistant Staphylococcus aureus isolates associated with a polymicrobial biofilm. Antimicrob Agents Chemother 51, 231-238 (2007).

7. Harbottle, H., Thakur, S., Zhao, S., White, D. G.: Genetics of antimicrobial resistance. Anim Biotechnol 17, 111-124 (2006).

8. Clinical and Laboratory Standards Institute: Performance Standards for Antimicrobial Susceptibility Testing; Twenty-Third Informational Supplement. CLSI document M100S23. Clinical and Laboratory Standards Institute, Wayne, 2013.

9. Oliveira, M., Monteiro, J. L., Rana, S., Vilela, C. L.: Antimicrobial resistance in Grampositive bacteria from Timorese River Buffalo (Bubalus bubalis) skin microbiota. Trop Anim Health Prod 42, 833-839 (2010).

10. Halpern, M. T., Schmier, J. K., Snyder, L. M., Asche, C., Sarocco, P. W., Lavin, B., Nieman, R., Mandell, L. A.: Meta-analysis of bacterial resistance to macrolides. J Antimicrob Chemother 55, 748-757 (2005).

11. Reyes, J., Hidalgo, M., Díaz, L., Rincón, S., Moreno, J., Vanegas, N., Castañeda, E., Arias, C. A.: Characterization of macrolide resistance in Gram-positive cocci from Colombian hospitals: A countrywide surveillance. Int J Infect Dis 11, 329-336 (2007).

12. Leclercq, R.: Mechanisms of resistance to macrolides and lincosamides: Nature of the resistance elements and their clinical implications. Clin Infect Dis 34, 482-492 (2002). 
13. Schmitz, F. J., Verhoef, J., Fluit, A. C., The Sentry Participants Group: Prevalence of resistance to MLS antibiotics in 20 European university hospitals participating in the European SENTRY surveillance programme. J Antimicrob Chemother 43, 783-792 (1999).

14. Schreckenberger, P., Ilendo, E., Ristow, K.: Incidence of constitutive and inducible clindamycin resistance in Staphylococcus aureus and coagulase-negative staphylococci in a community and a tertiary care hospital. J Clin Microbiol 42, 2777-2779 (2004).

15. Spiliopoulou, I., Petinaki, E., Papandreou, P., Dimitracopoulos, G.: erm(C) is the predominant genetic determinant for the expression of resistance to macrolides among methicillinresistant Staphylococcus aureus clinical isolates in Greece. J Antimicrob Chemother 53, 814-817 (2004).

16. Monroe, S., Polk, R.: Antimicrobial use and bacterial resistance. Curr Opin Microbiol 3, 496-501 (2000).

17. Sayah, R. S., Kaneene, J. B., Johnson, Y., Miller, R.: Patterns of antimicrobial resistance observed in Escherichia coli isolates obtained from domestic- and wild-animal fecal samples, human septage and surface water. Appl Environ Microbiol 71, 1394-1404 (2005).

18. Salyers, A., Shoemaker, N. B.: Reservoirs of antibiotic resistance genes. Anim Biotechnol 17, 137-146 (2006). 